Service social

\title{
L'identité professionnelle de travailleurs sociaux en devenir : de la formation à la pratique
}

\author{
Josianne Crête, Annie Pullen Sanfaçon et Isabelle Marchand
}

Volume 61, numéro 1, 2015

Être reconnu et protégé

URI : https://id.erudit.org/iderudit/1033739ar

DOI : https://doi.org/10.7202/1033739ar

Aller au sommaire du numéro

Éditeur(s)

École de service social de l’Université Laval

ISSN

1708-1734 (numérique)

Découvrir la revue

Citer cet article

Crête, J., Pullen Sanfaçon, A. \& Marchand, I. (2015). L’identité professionnelle de travailleurs sociaux en devenir : de la formation à la pratique. Service social, 61(1), 43-55. https://doi.org/10.7202/1033739ar
Résumé de l'article

La transition de la formation universitaire en service social à la pratique représente un moment important dans le processus de structuration de l'identité professionnelle. L'article présente les résultats issus d'une recherche qualitative longitudinale, qui s'est intéressée à ce processus dans cette période de transition. Selon un cadre interactionniste symbolique, ce processus est modélisé en trois moments qui concernent à tour de rôle : 1) l'acquisition de connaissances et de compétences professionnelles, 2) les enjeux d'autonomie et de reconnaissance professionnelles, et 3) la multiplicité des identités professionnelles en travail social. Par la suite, les résultats sont mis en dialogue avec les écrits scientifiques et, en conclusion, sont identifiées des pistes de réflexion. 


\title{
L'identité professionnelle de travailleurs sociaux en devenir : de la formation à la pratique
}

\author{
CRÊTE, Josianne \\ PULLEN SANFAÇON, Annie \\ MARCHAND, Isabelle
}

\begin{abstract}
RÉSUMÉ
La transition de la formation universitaire en service social à la pratique représente un moment important dans le processus de structuration de l'identité professionnelle. L'article présente les résultats issus d'une recherche qualitative longitudinale, qui s'est intéressée à ce processus dans cette période de transition. Selon un cadre interactionniste symbolique, ce processus est modélisé en trois moments qui concernent à tour de rôle : 1) l'acquisition de connaissances et de compétences professionnelles, 2) les enjeux d'autonomie et de reconnaissance professionnelles, et 3) la multiplicité des identités professionnelles en travail social. Par la suite, les résultats sont mis en dialogue avec les écrits scientifiques et, en conclusion, sont identifiées des pistes de réflexion.
\end{abstract}

Mots-clés : identité professionnelle, travail social, formation, éthique.

\begin{abstract}
The transition from university education to practice is an important moment in the process of structuring professional identity. The article presents the results of a qualitative longitudinal research project that questioned the process of structuring professional identity of social workers during this transition. Drawing from symbolic interactionism as theoretical framework, this process is conceptualised in three moments: 1) the acquisition of professional knowledge and skills, 2) the professional independence and recognition issues, and 3) the multiplicity of professional identities in social work. After examining these moments, they are discussed through scientific literature, which enables targeting further research needs.
\end{abstract}

Key words: professional identity, social work, education, ethics. 


\section{INTRODUCTION}

Au Québec, comme ailleurs dans le monde, l'organisation des services sociaux est de plus en plus axée sur leur technicisation et leur standardisation (Spolander et al., 2011). Les tâches et les rôles des travailleurs sociaux en sont de facto modifiés, remettant ainsi en question certains aspects de leur identité professionnelle (Hotho, 2008). Or, avoir une identité professionnelle forte permet de résister à la « rationalisation » de l'offre de services et aux modes de gestion axés sur les résultats, lesquels entrent en contradiction avec les orientations du travail social, centrées sur la relation à l'usager (OTSTCFQ, 2012). De plus, une solide identité professionnelle reste essentielle à la pratique éthique des travailleurs sociaux (PullenSansfaçon, 2011). Toutefois, pour arriver à se construire une identité professionnelle forte, les travailleurs sociaux doivent relever le défi suivant : intégrer l'identité professionnelle du travail social d'une façon cohérente avec leur identité sociale - qui ils sont comme personne -, tout en tenant compte de leur contexte de travail (Osteen, 2011). Cet exercice est complexifié de deux façons. D'abord, en raison des tensions qui se trouvent au sein de l'identité professionnelle du travail social. Blewett, Lewis et Tunstill (2007) regroupent ces tensions selon cinq axes : 1) évaluer ou offrir des services ; 2) faire de la prévention ou de la protection ; 3) intervenir dans la communauté ou en établissement ; 4) faire de la défense de droits visant le changement social ou de la thérapie visant le changement individuel ; et 5) mettre en application des services ou gérer les services offerts par d'autres. Ensuite, puisque le cadre organisationnel joue un rôle central dans la construction identitaire, les changements constants dans l'organisation des services compliquent aussi cette construction (Cnaan et Dichter, 2008). L'enjeu apparaît ainsi considérable pour les travailleurs sociaux puisqu'ils doivent articuler leur identité sociale à l'identité professionnelle en tension du travail social, et ce, dans un contexte en changement constant.

Il est généralement entendu que la formation initiale facilite l'articulation de ce défi identitaire, car elle est reconnue comme premier mode de socialisation professionnelle, contribuant de manière significative à la construction de l'identité professionnelle (Wiles, 2013). En revanche, l'entrée sur le marché du travail peut impliquer une renégociation de l'identité professionnelle développée durant la formation (McSweeney, 2011). En l'occurrence, il devient pertinent de se demander comment se développe et évolue l'identité professionnelle entre la fin de la formation universitaire en travail social et l'entrée sur le marché du travail ? Quels sont les aspects permettant de consolider l'identité professionnelle durant cette importante transition ?

L'article présente les résultats d'un projet de recherche explorant le processus de structuration de l'identité professionnelle de travailleurs sociaux dans leur passage de la formation à la pratique. Après avoir présenté le cadre théorique et méthodologique de cette étude, les résultats seront détaillés en trois sections, concernant chacune un moment du processus identitaire ayant émergé des données recueillies, soit: 1) l'acquisition de connaissances et de compétences professionnelles; 2) les enjeux d'autonomie et de reconnaissance professionnelles; et 3) la multiplicité des identités professionnelles en travail social. Ces résultats seront ensuite discutés à la lumière des connaissances théoriques portant sur le sujet, pour conclure en soulevant quelques pistes de réflexion. 


\section{CAdRes THÉORIQUe et MÉthodologiQue}

Le concept d'identité professionnelle se rapporte à la fois aux individus - collectivement ou individuellement - et aux groupes professionnels auxquels ils se rattachent. En ce sens, l'identité professionnelle est comprise comme «le résultat à la fois stable et provisoire, individuel et collectif, subjectif et objectif, biographique et structurel, des divers processus de socialisation qui, conjointement, construisent les individus et définissent les institutions » (Dubar, 2000, p. 109). Ici, c'est principalement le processus de structuration de l'identité professionnelle individuelle qui est étudié chez les nouveaux travailleurs sociaux. Dans ce processus, l'individu intègre graduellement les caractéristiques de l'identité professionnelle de son groupe d'appartenance - savoirs, valeurs, etc. Toutefois, il ne fait pas que ça, il les sélectionne, les réélabore et les réinterprète aussi subjectivement, jouant ainsi un rôle actif dans la construction de son identité professionnelle individuelle (Granja, 2008). Cette construction identitaire se modifie ensuite selon les contextes socioprofessionnels dans lesquels elle s'exprime (Chouinard et Couturier, 2006), puisque l'interaction entre les facteurs individuels et environnementaux est primordiale dans ce processus (Mathys, 2012). Cela est cohérent avec le cadre théorique interactionniste symbolique privilégié ici (Strauss, 1992).

C'est donc en faisant leurs les bases de l'identité de leur profession que les travailleurs sociaux construisent leur identité professionnelle individuelle, notamment en intégrant les valeurs propres au travail social (Dubar, 2010). Le terme valeur renvoie ici à une croyance forte intégrée par l'individu et qui agit comme référence pour juger ce qui est «bien ou mal » (Pullen-Sansfaçon et Cowden, 2012). Une valeur peut en être une « de préférence », si elle n'a que peu d'impact sur les actions de l'individu, ou en être une de « référence », qui guide alors les actions (Paquette, 1982). Sous cet angle axiologique, une identité professionnelle peut être qualifiée de forte lorsqu'un travailleur social intègre les valeurs du travail social ${ }^{1}$ comme valeurs de référence, ces dernières guidant alors ses actions et pratiques d'intervention.

Sur le plan méthodologique, une étude qualitative longitudinale étalée sur trois ans a été amorcée. Des trois phases prévues, la dernière reste à réaliser. À la première phase, un échantillon non aléatoire a été constitué de volontaires recrutés par un courriel envoyé à tous les étudiants inscrits en dernière année du baccalauréat en travail social de l'Université de Montréal, de l'Université du Québec à Montréal (UQÀM) et de l'Université du Québec en Outaouais (UQO). Treize participants ont alors été rencontrés en entrevue semi-dirigée pour comprendre l'état de leur identité professionnelle et son processus de structuration pendant la formation universitaire. Un guide d'entretien ciblait des thèmes à aborder, tout en laissant place à l'émergence de nouveaux thèmes. La deuxième phase visait à comprendre l'état de l'identité professionnelle et son développement six mois après l'arrivée sur le marché du travail. À cette étape, huit des participants initiaux ont été rencontrés à nouveau pour approfondir des thèmes ressortis de l'analyse des données de la première phase et des écrits portant sur le sujet. Ces

1. Par exemple, le respect de la dignité, le droit des personnes, leur autonomie et leur autodétermination, et la promotion de la justice sociale (OTSTCFQ, 2012). 
participants ont été ciblés dans une logique d'échantillonnage théorique, et ce, dans un souci de diversification des profils (sexe, âge, expérience, etc.) (Tableau 1).

TABLEAU 1 : PROFIL DES RÉPONDANTS AYANT PARTICIPÉ AUX DEUX PHASES (N=8)

\begin{tabular}{|l|c|c|r|}
\hline Pseudonyme & $\hat{\text { Âge }}$ & Sexe & Milieu de pratique (stage, emploi, expérience passée) \\
\hline Caroline & $22-25$ & $\mathrm{~F}$ & Stage et emploi au public / Expérience dans le communautaire \\
\hline Éric & $18-21$ & $\mathrm{M}$ & Stage et emploi au public / Expérience dans le communautaire \\
\hline Jérémie & $26-29$ & $\mathrm{M}$ & Stage au public / Emploi dans le communautaire \\
\hline Julien & $26-29$ & $\mathrm{M}$ & Stage et emploi au public / Expérience dans le communautaire \\
\hline Laure & $22-25$ & $\mathrm{~F}$ & Stage au public / Emploi dans le communautaire / \\
& & & Expérience au public et dans le communautaire \\
\hline Mélanie & $30-35$ & $\mathrm{~F}$ & Stage et emploi au public \\
\hline Nadine & $22-25$ & $\mathrm{~F}$ & Stage et emploi au public \\
\hline & $30-35$ & $\mathrm{~F}$ & Stages au public et dans le communautaire / Emploi au public \\
\hline & & & \\
\hline
\end{tabular}

Les entrevues ont été transcrites intégralement. Dans une logique inductive, les transcriptions ont d'abord été l'objet d'un codage ouvert, ce qui demande de rester fidèle aux propos tenus par les participants. Ensuite, la technique de la comparaison constante a permis de mettre en discussion ces codes entre eux, ainsi qu'avec les données empiriques et scientifiques, pour les regrouper en catégories (codage axial), représentant un niveau d'abstraction plus élevé (Guillemette et Lapointe, 2012). À cette étape, les données scientifiques ont été utilisées comme concepts sensibilisateurs afin de nourrir la théorisation émergeant des données empiriques (Guillemette et Luckerhoff, 2009). Tout au long de l'analyse, une attention a été portée à la fois à l'évolution de l'identité professionnelle des répondants ayant participé aux deux phases d'entrevues (analyse verticale), tout en questionnant les similitudes et les différences entre les cas étudiés (analyse horizontale).

Le présent article met l'accent sur les résultats ressortis de l'analyse horizontale des entrevues réalisées auprès des huit participants rencontrés aux deux phases - soit un total de 16 entrevues - sur une période de 18 mois. Inspirée par la théorie ancrée (grounded theory) (Glaser et Strauss, 1967), notre démarche analytique vise à produire un modèle théorique 
émergeant. Dans cette perspective, des catégories conceptualisantes ont été élaborées en vue de désigner les phénomènes en jeu (Paillé et Muchielli, 2012). II s'agit ici des processus de construction de l'identité professionnelle, déclinés en trois temps, entre la fin du baccalauréat en travail social et l'entrée sur le marché de l'emploi.

\section{PRÉSENTATION DES RÉSULTATS}

Les trois moments clés dans le processus de structuration de l'identité professionnelle des participants concernent: 1) l'acquisition de connaissances et de compétences professionnelles; 2) les enjeux d'autonomie et de reconnaissance professionnelles; et 3) la multiplicité des identités professionnelles en travail social. Chacun de ces moments ne représente pas en soi une étape à franchir, mais plutôt des « états d'être » s'interinfluençant dans un processus de construction identitaire. Ces états ne sont pas mutuellement exclusifs ; ils s'imbriquent, sans systématiquement découler l'un de l'autre. II s'agit donc d'un mouvement processuel, interactif et circulaire.

\section{Connaître et comprendre}

Le premier moment mis en lumière dans la structuration de l'identité professionnelle des participants concerne la connaissance et la compréhension de ce qui est nécessaire pour « être travailleur social ». Pendant leur formation initiale, leur stage de formation et leurs premières expériences de travail dans le domaine, les participants acquièrent une panoplie de connaissances et de compétences. La majorité évoque en ce sens avoir acquis « un bon bagage théorique ». Que ce soit sur les plans du savoir, du savoir-dire, du savoir-faire ou du savoir-être, ces savoirs restent en évolution puisque les répondants mentionnent continuer à s'outiller pour mieux connaître l'intervention, la culture organisationnelle, ainsi que les clientèles desservies.

[Au bac], on comprend c'est quoi l'intervention, on est capable d'expliquer c'est quoi, mais [...] faut arriver devant [...]. C'est vraiment là que tu mets en pratique ce que tu as pu apprendre, mais aussi, tu apprends beaucoup sur le tas. (Éric, phase 2)

Plus encore, les connaissances ne sont pas acquises «passivement »; les participants réinterprètent aussi les savoirs transmis à la lumière de leur identité sociale. Cette réinterprétation participe au processus de construction de leur identité professionnelle, dans une recherche de cohérence entre le « soi professionnel » et le « soi personnel ». Par exemple, les propos de Laure (phase 2) rappellent que les expériences professionnelles, positives ou négatives, sont filtrées à travers l'identité sociale qu'elle s'était jusqu'ici construite, où « ce qu'elle est comme personne » entre en dialogue avec ses nouvelles expériences et connaissances, structurant ainsi son identité professionnelle.

Je me souviens [de mon stage de technique en travail social fait à la Direction de la protection de la jeunesse (DPJ)], je n'avais pas nécessairement aimé ça, mais je n'étais pas fermée à l'idée de retourner éventuellement. Je me disais que, peutêtre [dans un autre programme,] ça allait plus être mon genre. Mais, finalement, 
c'est comme dans le fondement de l'affaire que ça ne me correspond pas. (Laure, phase 2)

Dans la même logique, les participants rapportent développer de nouveaux savoirs par leur expérience pratique. Ainsi, le processus de structuration de leur identité professionnelle est traversé non seulement par la réinterprétation de savoirs à travers de nouvelles expériences, mais aussi par le développement de nouveaux savoirs pratiques et leur concrétisation dans un milieu organisationnel. Soulignons également que le passage de l'acquisition à la réinterprétation de savoirs, puis au développement de savoirs pratiques, s'appuie sur le renforcement de la confiance en soi professionnelle. En effet, la majorité des participants (6 sur 8) rapporte que la confiance en soi est centrale dans le processus de structuration de l'identité professionnelle. C'est en ce sens que Caroline (phase 1) rappelle que lorsque «ton identité est forgée [...], tu es capable de l'affirmer », par exemple, dans le cadre d'une réunion d'équipe.

À ce sujet, cette dernière illustre très bien, à la phase 2, la circularité entre acquisition de savoirs, confiance en soi, identité professionnelle et développement de savoirs. Pour elle, une meilleure connaissance d'une problématique donnée et une plus grande expérience lui procureraient une plus grande confiance en soi, ce qui lui permettrait de consolider son identité professionnelle et, en cela, de mieux connaître ses capacités d'intervenante.

[Pour que mon identité professionnelle se solidifie,] il faudrait peut-être que je me spécialise dans quelque chose [...]. Peut-être que j'en connaîtrais un peu plus sur un sujet et qu'en travaillant là-dedans, je me sentirais peut-être plus outillée et plus en confiance. [...] On dirait que je n'ai pas encore assez d'expérience ou je n'ai pas encore assez essayé de choses pour vraiment avoir une confiance et savoir ce que je veux faire et ce que je suis capable. (Caroline, phase 2)

En fait, ceux qui se montrent moins confiants face aux savoirs pratiques qu'ils ont développés rapportent avoir une identité professionnelle moins forte que les autres. En d'autres mots, c'est en prenant confiance en soi professionnellement que les participants consolident leur identité professionnelle et qu'ils arrivent à jouer un rôle de plus en plus (pro)actif dans le développement et la réinterprétation de savoirs, ainsi que dans la compréhension de ce que c'est qu'« être travailleur social ». Pour plusieurs, ce sont en outre les expériences « terrain » qui permettent « de prendre du galon » comme l'évoque Éric (phase 2) et, ce faisant, de mieux comprendre le contexte d'intervention et d'agir en conséquence.

La structuration de l'identité professionnelle semble donc tributaire de la confiance en soi professionnelle de l'individu. À son tour, cette confiance est renforcée par l'acquisition et le développement de différents savoirs acquis dans la pratique. Cette circularité entre confiance en soi et savoirs participe à la structuration de l'identité professionnelle, aussi bien durant la formation pratique - le stage dont chacun tire des expériences différentes - qu'à l'entrée sur le marché de l'emploi. 


\section{Être autonome et reconnu}

Le deuxième moment du processus de structuration de l'identité professionnelle des travailleurs sociaux concerne les enjeux d'autonomie et de reconnaissance professionnelles. En acquérant plus de confiance en eux et en leurs savoirs, ils développent corolairement une plus grande autonomie dans l'intervention. Conséquemment, leur identité professionnelle s'en trouve aussi consolidée. Par exemple, lorsque questionné au sujet de ce qui l'a aidé à développer davantage son identité professionnelle, Éric (phase 2) répond :

On demande que je sois autonome, surtout dans le contexte où tout le monde est débordé. Et [...] ça me plaît. Je n'ai pas envie de me faire materner et « checker » par tout le monde, et de faire des choses que quelqu'un me dit de faire de cette manière-là. Au bout de la ligne, je finis par le faire, mais je me l'approprie [...]. Ça, je trouve que c'est vraiment quelque chose qui m'a aidé. (Éric, phase 2)

Éric se sent non seulement assez confiant pour s'approprier les prérequis à son intervention, mais l'autonomie qu'il en retire contribue aussi à affermir son identité professionnelle. En fait, la consolidation de l'identité professionnelle semble nécessiter le délaissement du «chapeau » d'étudiant ou de travailleur social en formation pour adopter graduellement celui de travailleur social, ce qui participe au développement de l'autonomie professionnelle, comme témoigne également Nadine (phase 1): «plus que je prends de l'autonomie, plus je me sens travailleuse sociale ».

Ainsi, gagner en autonomie donne le sentiment de faire dûment partie du groupe d'appartenance des travailleurs sociaux. Plusieurs participants y parviennent d'ailleurs, ce qui s'observe dans leur discours par le passage du « eux, les travailleurs sociaux » au « nous, les travailleurs sociaux ». Toutefois, se joue ici un réel enjeu d'autonomie, où un encadrement trop serré ou prolongé dans le temps peut être ressenti comme un frein au développement de l'autonomie, alors que, à l'inverse, la grande liberté laissée par une absence d'encadrement donne l'impression d'une trop grande autonomie et peut créer un sentiment d'isolement. Par exemple, Nadine (Phase 2) ressent qu'elle n'a pas eu assez d'autonomie en début d'emploi, expérience qui lui fait apprécier d'en avoir davantage ultérieurement. De son côté, Caroline exprime plutôt que le manque de suivis cliniques et le travail en «chacun pour-soi » ne permettent pas une réelle intégration dans l'équipe de travail.

L'autonomie se jouant dans le lien avec autrui - superviseurs, supérieurs, collègues travailleurs sociaux et d'autres disciplines -, il importe de souligner qu'autrui joue aussi un rôle de soutien permettant le renforcement de l'identité professionnelle. Ce soutien permet de répondre à au moins deux besoins des nouveaux intervenants: d'abord, la transmission de savoirs, ensuite la recherche de reconnaissance. En fait, au fur et à mesure que se structure l'identité professionnelle, l'enjeu de reconnaissance gagne en importance.

Quand tu te fais complimenter par un juge sur la qualité de ton travail, ou un avocat ou tes collègues [...] ça vient te recrinquer un peu ! [...] Ça aussi, ça a été un facteur qui m'a soutenu, la reconnaissance du milieu. (Éric, phase 2) 
Toutefois, tous ne vivent pas des expériences positives de reconnaissance. Par exemple, Patricia (phase 2) a vu ses compétences délégitimées par une inspection professionnelle réalisée quelques semaines après son entrée en poste. Celle-ci s'est conclue par : « dans leurs mots, j'étais une incompétente, je manquais de compétences, je mettais ma clientèle en danger ». Patricia fait un récit très émotif de cette inspection et de ce qui s'en est suivi: l'obligation de suivre des formations de mise à niveau. Ici, il importe de souligner le rôle central qu'a joué sa directrice en lui offrant soutien et reconnaissance. Cette dernière l'a « vraiment rassurée dans [s]es compétences », ce qui a contribué à structurer positivement son identité professionnelle de travailleuse sociale. C'est donc en combinant sa résilience à la reconnaissance de son milieu qu'elle a réussi, malgré l'expérience de déqualification vécue, à se sentir compétente, voire à renforcer son identité professionnelle et, in fine, à se sentir « bonne en intervention ».

Les résultats montrent ainsi qu'il existe un lien étroit et processuel entre la consolidation de l'identité professionnelle, l'autonomie professionnelle et la présence de reconnaissance dans le milieu du travail. Précisons finalement que les enjeux d'autonomie et de reconnaissance mettent en scène deux désirs en tension : d'un côté, le désir d'autonomisation nécessite de mettre autrui à distance et, de l'autre, le désir de reconnaissance exige la proximité à autrui. L'articulation de ces deux pôles apparaît essentielle au développement de la confiance en soi professionnelle, elle-même nécessaire à la consolidation de l'identité professionnelle.

\section{Consolidation de quelle identité professionnelle ?}

Nous l'avons évoqué, c'est d'abord par l'acquisition, la (ré)interprétation et le développement de savoirs que les participants solidifient leur confiance en soi pour développer une autonomie professionnelle, qu'ils souhaitent ensuite voir reconnue par autrui. Ce processus permet la consolidation de l'identité professionnelle qu'ils ont commencé à développer pendant leur formation initiale. Les résultats montrent à cet égard que l'identité professionnelle que les participants se construisent est loin d'être uniforme. C'est ce qui nous amène à penser que le troisième moment du processus de structuration identitaire est la négociation - et parfois la résolution - des tensions présentes au sein de l'identité professionnelle du travail social dans l'appropriation individuelle de cette identité de groupe.

En fait, les participants déclinent une panoplie de caractéristiques qui définissent - ou devraient, selon eux, définir - l'identité professionnelle du groupe du travail social, dont certaines entrent en compétition. On dénote par exemple des tensions entre une vision fonctionnaliste du travail social et une vision plus structurelle de la profession. Concrètement, les tensions entre ces visions demandent de faire des choix entre les valeurs et les principes qui constitueront l'identité du travail social et qui seront intégrés individuellement. Cela s'exprime entre autres en décidant de devenir, ou non, membre de leur ordre professionnel, ainsi que par le titre qu'ils utilisent pour désigner leur identité professionnelle. Le terme d' « intervenant » et « travailleur social » est utilisé par plusieurs, mais aussi celui de « militante » (Laure, phase 2). Le choix du titre utilisé n'est donc pas anodin, mais vise à se rattacher à l'un ou à l'autre des paradigmes en tension dans le champ théorique du travail social. Choisir de se 
rattacher à l'une ou à l'autre de ces visions devient toutefois un défi à mettre en action dans le milieu de travail, particulièrement lorsque l'emploi occupé est dans le réseau public.

Il y a ce petit côté revendicateur qui m'habite [...], c'est comme ça que je définis le travail social. À mon sens, c'est notre rôle de le faire et, dans les sphères du travail [social] qui me sont données [...] je ne retrouve pas cette liberté-là d'expression. (Laure, phase 2)

Visant ainsi à s'éloigner de la vision technicisée, voire fonctionnaliste du travail social, mise généralement de l'avant par le réseau public, Laure choisi, après son stage à la DPJ, d'œuvrer dans le milieu communautaire, là où elle trouve davantage de cohérence entre sa conception du travail social et la culture organisationnelle du milieu de pratique.

Ce constat permet de souligner qu'au moins deux façons de définir l'identité professionnelle du travail social cohabitent dans la pratique. Conséquemment, ces résultats montrent qu'il n'y aurait pas une identité univoque du travail social, intégrée et déployée de façon homogène par l'ensemble des travailleurs sociaux, mais plutôt une diversité d'identités professionnelles, rattachées à divers univers théoriques ou visions de la praxis. C'est cette polymorphie de l'identité professionnelle du travail social qui apparaît saillante au troisième moment du processus de structuration de l'identité professionnelle de nouveaux travailleurs sociaux.

\section{DISCUSSION}

Les résultats de l'étude présentent des convergences et des divergences au regard des écrits sur le sujet. Tout d'abord, soulignons que deux classifications des savoirs ont dû être utilisées pour illustrer les résultats obtenus. La première est celle habituellement utilisée en travail social - savoir, savoir-dire, savoir-faire et savoir-être - où les savoirs se distinguent par l'objet sur lequel ils portent. La deuxième classification distingue plutôt les savoirs selon leur mode de production : les savoirs théoriques construits grâce à des réflexions conceptuelles, les savoirs pratiques bâtis par la pratique d'activités humaines, et les savoirs existentiels acquis par la socialisation (Galvani, 1999). Se centrer ainsi sur le mode de production des savoirs permet de comprendre si l'individu se les approprie ou les développe, mettant ici en évidence que l'identité professionnelle se structure par une mise en action, une autonomisation grandissante dans l'appropriation, l'interprétation et le développement de savoirs.

Il est aussi intéressant de souligner que Dubar (2000) propose que les savoirs scientifiques - qu'il généralise aux savoirs spécialisés - donnent du pouvoir aux professionnels qui les détiennent, leur permettant une forme d'autonomie professionnelle. C'est ce qui leur permet ensuite d'être reconnus et de structurer une identité professionnelle positive (Honneth, 2004). Les liens entre ces divers concepts sont corroborés par les résultats obtenus. Toutefois, ceux-ci montrent l'importance de la confiance en soi - une caractéristique qui renvoie davantage à la subjectivation de soi et de son identité professionnelle - dans la façon dont sont liés ces concepts. Ainsi, ce n'est pas uniquement l'intégration de savoirs scientifiques qui donne accès à l'autonomie professionnelle, puis à la reconnaissance permettant le développement d'une identité professionnelle réussie, mais aussi la confiance en ces savoirs et en ses aptitudes à les déployer. 
Ensuite, Provencher (2010, p. 33) souligne que la structuration de l'identité professionnelle se fait dans « la rencontre entre le processus d'identification (sentiment d'appartenance) et celui de l'identisation (affirmation de soi) ». Ce processus dialogique correspond à nos résultats selon lesquels l'identité professionnelle se structure à travers un processus d'autonomisation de l'individu. Par contre, pour cet auteur, l'identisation sous-entend que c'est l'identité sociale de l'individu qui influencera la façon dont il s'identifiera à son groupe d'appartenance, ce qui ne correspond pas précisément à l'ensemble des données obtenues. En effet, bien que les participants rapportent que leur identité sociale influe sur la structuration de leur identité professionnelle, ils soulignent que d'autres éléments y participent aussi. En fait, leurs expériences antérieures, ainsi que différents facteurs environnementaux contribuent à la configuration de leur identité professionnelle. Ces facteurs sont, par exemple, la structure de leur établissement d'embauche, les valeurs prônées par le milieu et par leurs collègues de diverses disciplines, ainsi que la réalité sociopolitique qui les entoure.

Enfin, le dernier élément qui mérite d'être souligné concerne l'adhésion à une seule interprétation de l'identité professionnelle du travail social. En fait, cette question fait écho au concept de «segment professionnel » proposé par Strauss (1992). Cette notion déconstruit l'idée qu'une profession endosse une identité professionnelle homogène ; elle se constituerait plutôt à travers des sous-groupes professionnels multiples, ce qui créait son hétérogénéité. Dans cette perspective, nos résultats montrent que le travail social englobe une multitude de façons de mettre en action une identité professionnelle, tel que le suggèrent également Blewett, Lewis et Tunstill (2007). En conséquence, tenir compte de ces multiples segments professionnels et des tensions internes en jeu apparaît nécessaire à la construction d'une identité professionnelle forte.

\section{ConClusion}

En résumé, les résultats suggèrent que le processus de structuration de l'identité professionnelle des travailleurs sociaux est dynamique, interactif et circulaire. Les aspects dynamiques et interactifs de ce processus viennent du fait que les trois moments décrits s'influencent mutuellement, sans pour autant dépendre l'un de l'autre. Ainsi, il ne s'agit pas d'un processus mécanique, qui se réalise en étapes bien définies. De plus, ces moments présentent une forme de circularité et, plus précisément, se déclinent en étapes itératives, où il faut d'abord acquérir des savoirs pour ensuite se confronter aux enjeux d'autonomie et de reconnaissance, ce qui permet de consolider une identité professionnelle cohérente avec son interprétation de l'identité professionnelle du groupe du travail social. Cette consolidation identitaire, médiatisée par le renforcement de la confiance en soi, appelle alors la révision des savoirs acquis et la recherche de nouveaux savoirs. Ce processus réactive les enjeux d'autonomie et de reconnaissance et, dans un effet d'aller-retour, suscite une réinterprétation de l'identité professionnelle en place.

Cela dit, plusieurs aspects abordés mériteraient davantage d'approfondissement. Par exemple, sur le plan méthodologique, vérifier la transférabilité des résultats auprès d'autres travailleurs sociaux ou d'autres groupes professionnels pourrait être intéressant, tout comme le fait de comparer la structuration de l'identité professionnelle dans d'autres champs du social. 
Par ailleurs, puisque les participants ont mentionné que leur milieu a une influence sur la structuration de leur identité, il apparaît pertinent de viser à mieux comprendre le rôle joué par le contexte organisationnel dans le développement de l'identité professionnelle des participants.

De la même façon, il serait intéressant d'approfondir les enjeux éthiques que soulève la multiplicité des identités professionnelles présentes dans la diversité des formes que prend l'identité professionnelle du travail social chez les répondants. À cet égard, certains auteurs (par exemple, RUFUTS, 1998) sous-entendent que l'absence d'une identité professionnelle collective unique - ou unifiée - est signe d'une faiblesse identitaire de la profession. Cette lacune rendrait les professionnels plus influençables par le contexte dans lequel ils évoluent, rendant leur pratique moins conforme aux attentes disciplinaires. En revanche, n'est-il pas possible que cette multiplicité d'identités professionnelles représente plutôt une richesse, tant sur le plan des pratiques d'intervention que pour la profession et le champ du travail social ? $\mathrm{Ou}$, au contraire, est-ce le résultat des politiques macroéconomiques et de leurs effets structurants sur les intervenants et les services (Spolander, Engelbrecht et Pullen-Sansfaçon, sous presse) ? Ainsi, bien loin d'être closes, de nombreuses questions identitaires et enjeux éthiques restent en suspens, notamment dans un contexte où les changements structurels qui ont lieu dans le système public posent une série de défis aux travailleurs sociaux d'aujourd'hui.

CRÊTE, Josianne

T.S., doctorante École de service social de l'Université de Montréal

PULlen SANFAÇON, Annie

B.Serv.Soc., PhD. Professeure agrégée

École de service social de l’Université de Montréal

MARCHAND, Isabelle

Doctorante, École de service social de 1'Université de Montréal,

\section{RÉFÉRENCES}

Blewett, J., J. Lewis et J. Tunstill (2007). The Changing Roles and Tasks of Social Work: A Literature Informed Discussion Paper, [Londres, General Social Care Council].

Chouinard, I., et Y. Couturier (2006). « Identité professionnelle et souci de soi en travail social », Nouvelles pratiques sociales, vol. 19, n 1, p. 176-182.

Cnaan, R.A., et M.E. Dichter (2008). «Thoughts on the Use of Knowledge in Social Work Practice », Research on Social Work Practice, vol. 18, n 4, p. 278-284.

Dubar, C. (2000). La socialisation. Construction des identités sociales et professionnelles. $3^{\mathrm{e}}$ éd., Paris, Armand Colin.

Dubar, C. (2010). La socialisation. Construction des identités sociales et professionnelles, $4^{\mathrm{e}}$ éd., Paris, Armand Colin. 
Galvani, P. (1999). « Fertilisation croisée des savoirs et ingénierie d'alternance socio-formative. Le programme de recherche-formation-action Quart Monde/Université », Revue française de pédagogie, $\mathrm{n}^{0} 128$, p. 25-34.

Glaser, B.G., et A.L. Strauss (1967). The Discovery of Grounded Theory: Strategies for qualitative research. New York, Aldine de Gruyter.

Granja, B. (2008). «Éléments de construction identitaire professionnelle des assistants de service social en formation », Travail-Emploi-Formation, nº 8, p. 21-38.

Guillemette, F., et J.-R. Lapointe, (2012). « Illustration d'un effort pour demeurer fidèle à la spécificité de la méthodologie de la théorisation enracinée (Grounded Theory) », dans J. Luckerhoff et F. Guillemette (dir.), Méthodologie de la théorisation enracinée. Fondements, procédures et usages, Québec, Presses de l'Université du Québec, p. 11-35.

Guillemette, F., et J. Luckerhoff (2009). « L'induction en méthodologie de la théorisation enracinée (MTE) », Recherches qualitatives, vol. 28, n² 2, p. 4-21.

Honneth, A. (2004). «La théorie de la reconnaissance: une esquisse », Revue du MAUSS, vol. 23, n 1, p. 133-136.

Hotho, S. (2008). «Professional identity-product of structure, product of choice: Linking changing professional identity and changing professions », Journal of Organizational Change Management, vol. 21, $\mathrm{n}^{\circ}$ 6, p. 721-742.

Mathys, C. (2012). Le rôle des nouvelles technologies dans le travail et la construction identitaire des journalistes politiques de la presse écrite, [Montréal], Mémoire de maîtrise. Département de communication, Université de Montréal.

McSweeney, F. (2011). « Student, Practitioner, or Both? Separation and Integration of Identities in Professional Social Care Education », Social Work Education, vol. 31, n 3, p. 364-382.

Mercier, C., et R. Mathieu (2000). « Le travail social : nouveaux enjeux, nouvelles pratiques », Nouvelles pratiques sociales, vol. $13, \mathrm{n}^{\circ} 1, \mathrm{p} .15-25$.

Paillé, P., et A. Mucchielli (2012). L'analyse qualitative en sciences humaines et sociales ( $3^{\mathrm{e}}$ éd.), Paris, Armand Colin.

Osteen, P.J. (2011). « Motivations, Values, and Conflict Resolution: Students' Integration of Personal and Professional Identities », Journal of Social Work Education, vol. 47, $\mathrm{n}^{\circ} 3$, p. 423-444.

OTSTCFQ (Ordre des travaillers sociaux et des thérapeutes conjugaux et familiaux du Québec) (2012). Référentiel des compétences des travailleuses sociales et des travailleurs sociaux, [s. I. ], OTSTCFQ.

Paquette, C., (1982), Analyse de ses valeurs personnelles. S'analyser pour mieux décider, Montréal, Québec/Amérique. 
Provencher, A. (2010). Développement de l'identité professionnelle d'enseignantes débutantes en contexte de relation mentorale, [Montréal], Mémoire de maîtrise, Département de psychopédagogie et andragogie, Université de Montréal.

Pullen-Sansfaçon, A. (2011). «Ethics and Conduct in Self-directed Groupwork: Some Lessons for the Development of a More Ethical Social Work Practice », Ethics and Social Welfare, vol. $5, n^{\circ} 4$, p. 361-379.

Pullen-Sansfaçon, A., et S. Cowden (2012). The Ethical foundations of social work, Londres, Routledge.

RUFUTS (Regroupement des Unités de Formation Universitaire en Travail Social) (1998). L'avenir de la profession du travail social : point de vue des universitaires, Montréal, Compte rendu d'une Journée de réflexion, récupéré de :

http://www.unites.uqam.ca/rufuts/Archives1998.html.

Spolander, G., Pullen-Sansfacon, A., Brown, M., et Engelbrecht, L. (2011). « Social work education in Canada, England and South Africa: A critical comparison of undergraduate programmes », International Social Work, vol. 54, n 6, p. 816-831.

Spolander, G., L. Engelbrecht et A. Pullen-Sansfaçon (sous presse). « Social Work and Macroeconomic Neoliberalism : Beyond social justice rhetoric », European Journal of Social Work.

Strauss, A. L. (1992). La trame de la négociation. Sociologie qualitative et interactionnisme, Paris, L'Harmattan.

Wiles, F. (2013). « "Not Easily Put Into a Box": Constructing Professional Identity », Social Work Education, vol. 32, n 7 , p. 854-866. 